\title{
Onset of convection in a rotating annulus with radial gravity and heating
}

\author{
A. Alonso ${ }^{\text {a }}$, M. Net ${ }^{\text {a }}$, I. Mercader ${ }^{\text {a }}$, E. Knobloch ${ }^{\text {b,* }}$ \\ ${ }^{a}$ Universitat Politècnica de Catalunya, Campus Nord. Mòdul B-4, 08034 Barcelona, Spain \\ ${ }^{\mathrm{b}}$ Department of Physics, University of California, Berkeley, CA 94720, USA
}

Received 10 November 1997; revised 30 March 1998; accepted 22 April 1998

\begin{abstract}
The onset of convection in fluids confined in a vertical rotating cylindrical annulus with radial gravity and heated from the inside is analyzed. Solutions to the eigenvalue problem are found numerically for flat lids and either no-slip or stress-free boundary conditions on these surfaces. Except in special cases occurring with the latter boundary conditions all critical modes precess in the rotating frame. The critical Rayleigh number and the corresponding precession frequency are computed for low-Prandtl number fluids and both types of boundary conditions as a function of the Taylor number, the radius ratio and the aspect ratio, and the critical modes identified and compared. (C) 1999 The Japan Society of Fluid Mechanics and Elsevier Science B.V. All rights reserved.
\end{abstract}

\section{Introduction}

Thermal convection driven by radial heating in an annulus rotating uniformly about its axis has long been studied as the simplest model of convection in stellar and planetary atmospheres and planetary interiors. Accurate laboratory experiments have been carried out by several authors to investigate different aspects of the dynamics resulting from a destabilizing thermal gradient perpendicular to rotation. These depend fundamentally on the direction of gravity. If gravity is parallel to the temperature gradient a motion-free conduction state is present and the observed dynamics result from instabilities of this state. In contrast if gravity acts in a direction that is orthogonal to the temperature gradient (and antiparallel to the rotation vector) the basic state consists of an axisymmetric thermal wind. These situations are referred to as barotropic and baroclinic, respectively. The former has been studied as a model for convection in the equatorial regions of the earth's interior using the centrifugal force to mimic radial gravity and heat input from the outside (Busse and Carrigan, 1974; Azouni et al., 1986), with emphasis on the Rossby waves generated

*Corresponding author. E-mail: knobloch@physics.berkeley.edu. 
by slanted top and bottom ends. Experimental studies of the baroclinic case have been much more numerous and help elucidate the dynamics of baroclinic waves at higher latitudes. Such studies have been carried out either with a free upper surface (Hide and Mason, 1975), or with a rigid lid (Fein and Pfeffer, 1976; Read et al., 1992), and focus on regimes with highly nonlinear vacillating or chaotic behavior. Both situations lead to nonaxisymmetric waves which drift in a preferred direction in the rotating frame. In the following, we shall refer to such waves as precessing waves.

The present paper seeks to shed light on the origin of precession in rotating systems. General theory (Knobloch, 1994) predicts that in such systems all nonaxisymmetric instabilities generically give rise to precessing states. This prediction is in apparent conflict with the early analytical work of Busse (1970) on the barotropic case with horizontal lids. In this work Busse used stress-free boundary conditions on the lids and noted the presence of a time-independent two-dimensional solution in the form of Taylor-Proudman columns. This solution is characterized by an exact balance between the pressure gradient and the Coriolis force. With slanted ends the Taylor-Proudman solution is no longer time independent and takes the form of slowly drifting Rossby waves. In this paper we show explicitly that precessing Taylor-Proudman columns are present in the barotropic problem even with horizontal lids, provided only that no-slip boundary conditions are employed. As noted by Knobloch (1994) and Alonso et al. (1995) these boundary conditions play a crucial role in that they prevent the existence of the two-dimensional Taylor-Proudman solution. With these boundary conditions the flow near the lids must necessarily be vortical and hence cannot be balanced by a pressure gradient. In fact, we show that this conclusion holds for any nonstress-free boundary conditions, in agreement with the abstract theory. These solutions are not to be thought of as Rossby waves, however, since the precession is not topographically induced. In general, any three-dimensional solution (i.e., a solution with nonzero axial and azimuthal wave numbers) will precess, and such states can be the preferred mode of convection even with stress-free boundary conditions at top and bottom (Alonso et al., 1995). In this paper we extend the calculations of Alonso et al. to no-slip boundary conditions, focusing on the preferred modes of convection for low Prandtl number fluids and their associated precession frequencies. In the limit of rapid rotation we expect convection to be nearly two-dimensional with departures from two-dimensionality confined to thinner and thinner Ekman boundary layers at the top and bottom plates. Since it is these boundary layers that are ultimately responsible for the precession of the Taylor-Proudman columns we expect the precession frequency to vanish in the infinite rotation limit. Similar conclusions were recently reached by Zhang and Greed (1997) in a study of a rotating annulus with uniform internal heating.

The remainder of the paper is organized as follows. In Section 2, we summarize the basic equations and the method used to solve them. In Section 3, we present (in two subsections) the results obtained for low and high rotation rates. The significance of our results is summarized in Section 4.

\section{The equations and numerical method}

The stability of the conduction state of a Boussinesq fluid that fills a cylindrical annulus of radius ratio $\eta=r_{i} / r_{o}$ and height $L$ rotating about its axis of symmetry is considered. The linearized equations, nondimensionalized using the gap width $d=r_{o}-r_{i}$ as lengthscale, $d^{2} / \kappa$ as timescale, where $\kappa$ is the 
thermal diffusivity, and $\Delta T \equiv T_{i}-T_{o}$ as temperature scale, are

$$
\begin{aligned}
& \sigma^{-1} \frac{\partial \boldsymbol{u}}{\partial t}=-\nabla p+\operatorname{Ra} \Theta \hat{\boldsymbol{e}}_{r}+\nabla^{2} \boldsymbol{u}-2 \Omega \times \boldsymbol{u} \\
& \frac{\partial \Theta}{\partial t}=-\frac{u}{r \ln \eta}+\nabla^{2} \Theta \\
& \nabla \cdot \boldsymbol{u}=0 .
\end{aligned}
$$

Here $\boldsymbol{u}=(u, v, w)$ is the velocity field, $\boldsymbol{g}=-g \hat{\boldsymbol{e}}_{r}$ is the gravitational acceleration, $\Theta$ denotes the departure of the temperature from its conduction profile $T(r) / \Delta T, T(r)=T_{i}+\Delta T \ln \left(r / r_{i}\right) / \ln \eta$, and $\Omega$ is the rotation rate expressed in viscous units. The Rayleigh, Prandtl and Taylor numbers are defined, respectively, by

$$
\mathrm{Ra}=\frac{\alpha \Delta T g d^{3}}{\kappa v}, \quad \sigma=\frac{v}{\kappa}, \quad \mathrm{Ta}=4 \Omega^{2},
$$

where $\alpha$ is the coefficient of thermal expansion and $v$ is the kinematic viscosity.

The problem is solved with no-slip and perfectly conducting boundary conditions on the lateral walls

$$
\boldsymbol{u}=\Theta=0 \quad \text { on } r=r_{i}, r_{o}
$$

and either stress-free or no-slip, thermally insulating boundary conditions at top and bottom:

$$
\begin{aligned}
& \frac{\partial u}{\partial z}=\frac{\partial v}{\partial z}=w=\frac{\partial \Theta}{\partial z}=0 \quad \text { on } z=0, \beta, \\
& u=v=w=\frac{\partial \Theta}{\partial z}=0 \quad \text { on } z=0, \beta .
\end{aligned}
$$

Here $\beta \equiv L / d$ is the inverse aspect ratio of the annulus.

Eqs. (1)-(3) are solved numerically using a potential formulation (Marqués et al., 1993) and the eigenfunction $\Psi \equiv(\boldsymbol{u}, \Theta)$ is expanded in the form

$$
\Psi(r, \phi, z, t)=e^{s t} \sum_{l m n} a_{l m n} T_{l}(r) f_{m}(z) \mathrm{e}^{\mathrm{in} \phi},
$$

where $s$ is the growth rate, $T_{l}(r)$ is the Tchebyshev polynomial of order $l$ that specifies the radial structure of the eigenfunction, and $f_{m}(z)$ represents a trigonometric function for boundary conditions (3a) and a Tchebyshev polynomial for boundary conditions (3b). The integers $(m, n)$ indicate the structure in the axial and azimuthal directions, respectively. Although the problem is nonseparable in $r, z$ when boundary conditions (3b) are used it can be divided into symmetric or antisymmetric problems with respect to mid-height. 


\section{Numerical results}

Almost all the results presented in this section correspond to a Prandtl number $\sigma=0.025$, but as far as the linear problem is concerned, all of them are qualitatively very similar to those found for other fluids of moderate $\sigma$ values, as will be seen below.

\subsection{Low rotation rates}

Fig. 1 shows the variation of the Rayleigh number at the onset of convection in a nonrotating annulus as a function of the radius ratio for both sets of boundary conditions. The remaining parameter values are indicated in the figure. The two parts of the figure look similar, with changes in the azimuthal structure of the dominant modes taking place at nearly identical values of $\eta$, indicating a strong constraining influence of the annular geometry. However, there are also differences. In case (3a) the dashed lines labelled $n=2,3, \ldots$ correspond to $m=0$ solutions without axial structure, i.e., to Taylor-Proudman columns. These solutions are independent of the inverse aspect ratio $\beta$. The $n=1$ Taylor-Proudman solution has a higher critical Rayleigh number and is not shown. Throughout most of the $\eta$ range considered these modes set in first. However, there are intervals in $0<\eta<0.3$ in which the first instability is to an $m=1, n=1$ state and not a Taylor-Proudman state. The existence of these solutions is a manifestation of the tendency of rolls to align themselves parallel to the shorter dimension of any container: large $\beta$ favors axisymmetric rolls while small $\beta$ favors columnar convection, other parameters being fixed. The three-dimensional states are found near the cross-over between these two types of states. In contrast, in case (3b) the corresponding solutions always have

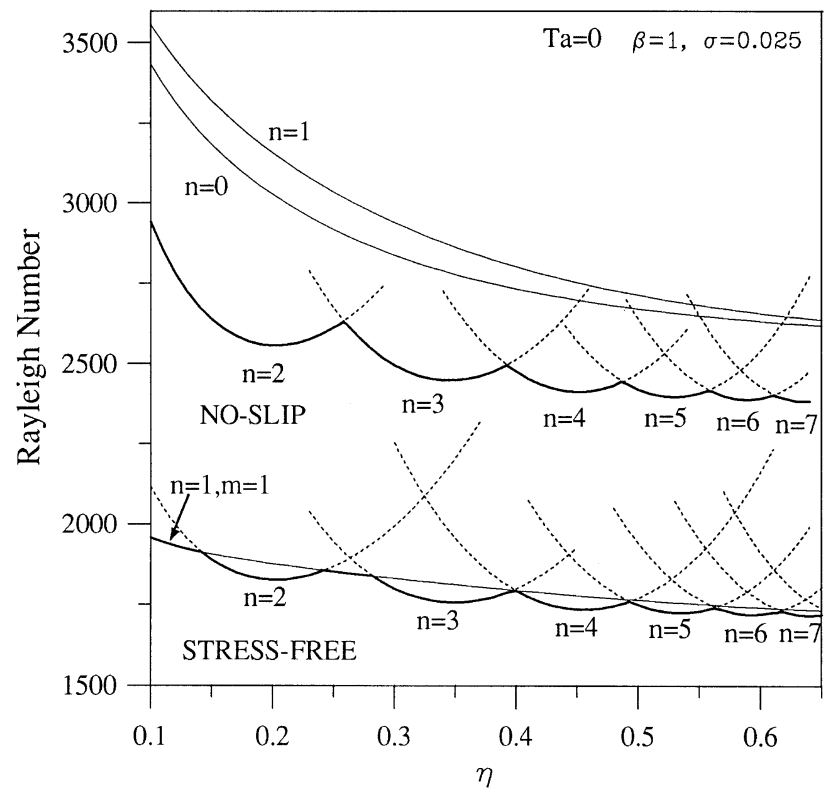

Fig. 1. Critical Rayleigh number as a function of radius ratio, $\eta$, for no-slip (upper panel) and stress-free (lower panel) boundary conditions on the lids. The heavy line indicates the envelope. The parameters are Ta $=0, \beta=1$ and $\sigma=0.025$. 


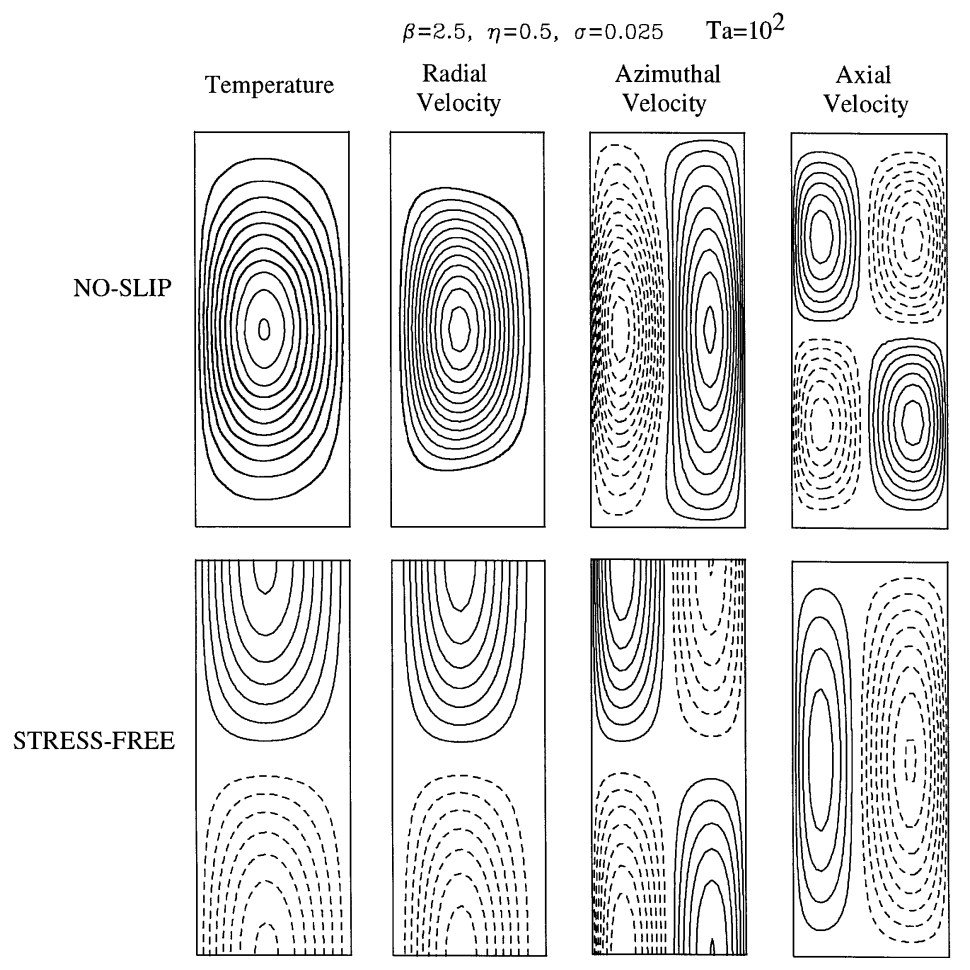

Fig. 2. Contour plots of the velocity components and temperature perturbation showing the axial structure of the dominant convection modes in the $(r, z)$ plane for $\mathrm{Ta}=10^{2}, \beta=2.5, \eta=0.5$ and $\sigma=0.025$ and stress-free and no-slip boundary conditions on the lids. The modes have opposite parities with respect to mid-height; both are three dimensional.

a nontrivial vertical structure which does depend on the value of $\beta$ (cf. Fig. 2). Because of the noslip boundary conditions all (nonaxisymmetric) critical modes are three-dimensional, and the critical Rayleigh numbers correspondingly higher than in the stress-free case. However, in both cases the dashed curves correspond to symmetric modes with respect to mid-height while the thin solid lines correspond to antisymmetric modes. Note that in the stress-free case an antisymmetric mode $(m=1)$ is sometimes preferred, while with no-slip boundaries the preferred modes are always symmetric, at least for moderate aspect ratios (cf. Fig. 2). In the former case the axial velocity near the outer wall is either in the direction of the rotation vector with a recirculation near the inner wall or vice versa, while in the latter the axial velocity vanishes at mid-height at all radii. We can think of the antisymmetric solutions as representing the type of flow seen with no-slip boundaries, but only in an annulus of half the full height. This is because the stress-free boundaries are of Neumann type and these allow one to construct solutions for a cylinder of twice the height by a simple reflection in one of the boundaries, i.e., the solution shown represents only half a wavelength of the full solution. In contrast, the solution depicted for the no-slip case represents a complete wavelength. Qualitatively, similar behavior occurs for all sufficiently small rotation rates provided $\beta \gtrsim 1$.

Fig. 3a, computed with the boundary conditions (3a), shows the critical Rayleigh number versus the (inverse) aspect ratio $\beta$ for the dominant modes when the rotation rate is slow $\left(\mathrm{Ta}=10^{2}\right)$. For fixed $\eta=0.5$ and $\beta \gtrsim 1.5$, the azimuthal wave number remains constant $(n=4)$ and only the axial 

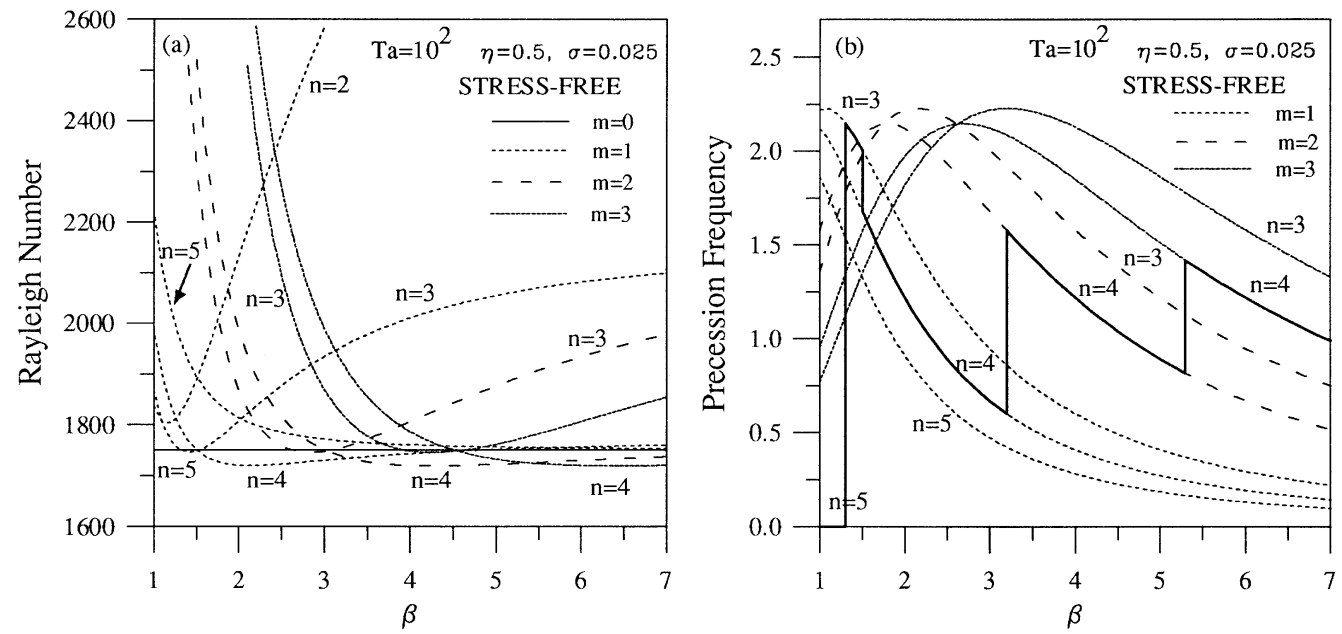

Fig. 3. (a) The critical Rayleigh number and (b) the corresponding precession frequency in viscous units as functions of the (inverse) aspect ratio, $\beta$, for stress-free boundary conditions on the lids and $\mathrm{Ta}=10^{2}$.
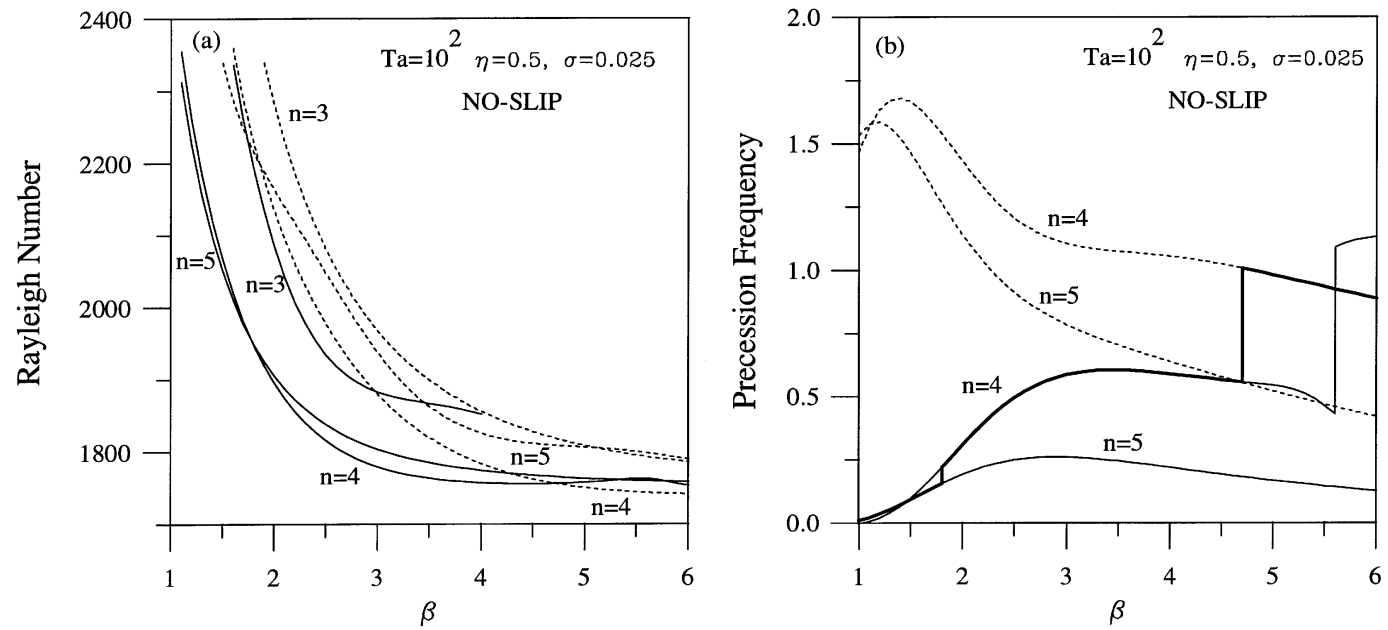

Fig. 4. (a) The critical Rayleigh number and (b) the corresponding precession frequency in viscous units as functions of the (inverse) aspect ratio, $\beta$, for no-slip boundary conditions on the lids and $\mathrm{Ta}=10^{2}$.

wave number increases when the length of the annulus increases. Because of their three-dimensional character (see Fig. 2) the precession frequency of these modes is nonzero, as shown (in viscous units) in Fig. 3b. The heavy line indicates the precession frequency of the dominant modes. Only for $\beta \lesssim 1.3$ does the dominant mode take the form of (nonprecessing) Taylor-Proudman columns, with azimuthal wave number $n=5$. The corresponding results for no-slip boundary conditions are shown in Fig. 4 plotted on the same scale as Fig. 3. Observe that the dominant mode is again an $n=4$ mode, this time for $\beta \gtrsim 1.8$; for sufficiently large $\beta$ the influence of the lids wanes and the asymptotic azimuthal wave number is determined by the radius ratio which has been kept 
unchanged. For smaller values of $\beta$ the dominant mode is again $n=5$; the small interval in $\beta$ with a three-dimensional $n=3$ state seen in Fig. 3 is now absent. Both the $n=4$ and the $n=5$ modes are three-dimensional (see Fig. 2) and hence precess, as indicated in Fig. 4b; for $\beta \gtrsim 3$ the precession frequencies are comparable to those found in the stress-free case. Note that there are, in fact, several different types of $n=4$ modes, with a transition between them near $\beta=4.6$ and near $\beta=5.6$ (Fig. 4). These modes differ in their axial structure, with solid and dashed lines indicating symmetric and antisymmetric modes, respectively. In each case the system selects a mode with a shorter axial wavelength; the first transition involves modes of opposite parity with respect to mid-height while the second involves a transition between two symmetric $n=4$ modes. The latter appears as a cusp in the thin solid curve labelled $n=4$ in Fig. 4a with a corresponding jump in frequency in Fig. 4b. We surmise that the observed transitions take place when the dissipation along the curved walls of the annulus exceeds that in the Ekman boundary layers at the lids. In contrast, the preferred solutions for $\beta \lesssim 1$ (not shown) take the form of Taylor-Proudman columns, and these are symmetric, with no nodes in the axial direction. Regardless of the boundary conditions these modes have azimuthal wave number $n=5$; as already mentioned such modes precess only in the no-slip case. For $\mathrm{Ta}=10^{2}, \eta=0.5$ this mode ceases to be the preferred one near $\beta=1.8$; however, for sufficiently large Taylor numbers it remains the dominant mode regardless of the value of $\beta$.

\subsection{High rotation rates}

The results presented in this section are for Taylor numbers high enough to have geophysical and astrophysical interest. Alonso et al. (1995) show in detail that in the stress-free case there is always, for any value of the parameters $\eta$ and $\beta$, a moderate rotation rate at which convection sets in as steady Taylor-Proudman columns whose azimuthal structure depends on $\eta$. Consequently, we have focused mainly on the more interesting no-slip case.

Fig. 5 displays the aspect ratio dependence of (a) the critical Rayleigh number and (b) the precession frequency in viscous units for small, $\sigma=0.025$, and moderate, $\sigma=0.7$, Prandtl numbers. The critical Rayleigh number, $\mathrm{Ra}_{\mathrm{c}}$, is independent of $\beta$ for boundary conditions (3a) because columnar convection is independent of the axial coordinate; in contrast with boundary conditions (3b) it decreases with increasing $\beta$, reaching asymptotically the stress-free result. This can also be seen in Fig. 5 b, which shows that the frequency tends to zero as $\beta \rightarrow \infty$, after first crossing the $\beta$-axis, i.e., for large enough $\beta$ (small enough aspect ratio) the precession is prograde $\left(\omega_{\mathrm{c}}<0\right)$. The exact location of the transition from retrograde-to-prograde precession depends both on the Prandtl number and the Taylor number. It is important to note that the critical Rayleigh numbers in Fig. 5a are essentially identical, with only the precession frequency, $\omega_{\mathrm{c}}$, showing any significant Prandtl number dependence (see Fig. 5b). This is a consequence of the fact that the precession frequency of these modes is relatively small.

In Fig. 6 we study the $\eta$-dependence of the critical Rayleigh number and the precession frequency of the dominant mode for no-slip boundary conditions, at fixed $\beta$. Fig. 6a resembles Fig. 1. The change in the wave number $n$ takes place at similar values of $\eta$, suggesting again the influence of the curvature of the lateral walls. Evidently, the radius ratio exerts a strong influence on the selected azimuthal wave number. In both cases we expect to observe, in the nonlinear regime, the phenomenon of "mode-jumping" with increasing Rayleigh number, as different azimuthal wave numbers become unstable. However, because of the strong Rayleigh number dependence of the neutral stability curves 

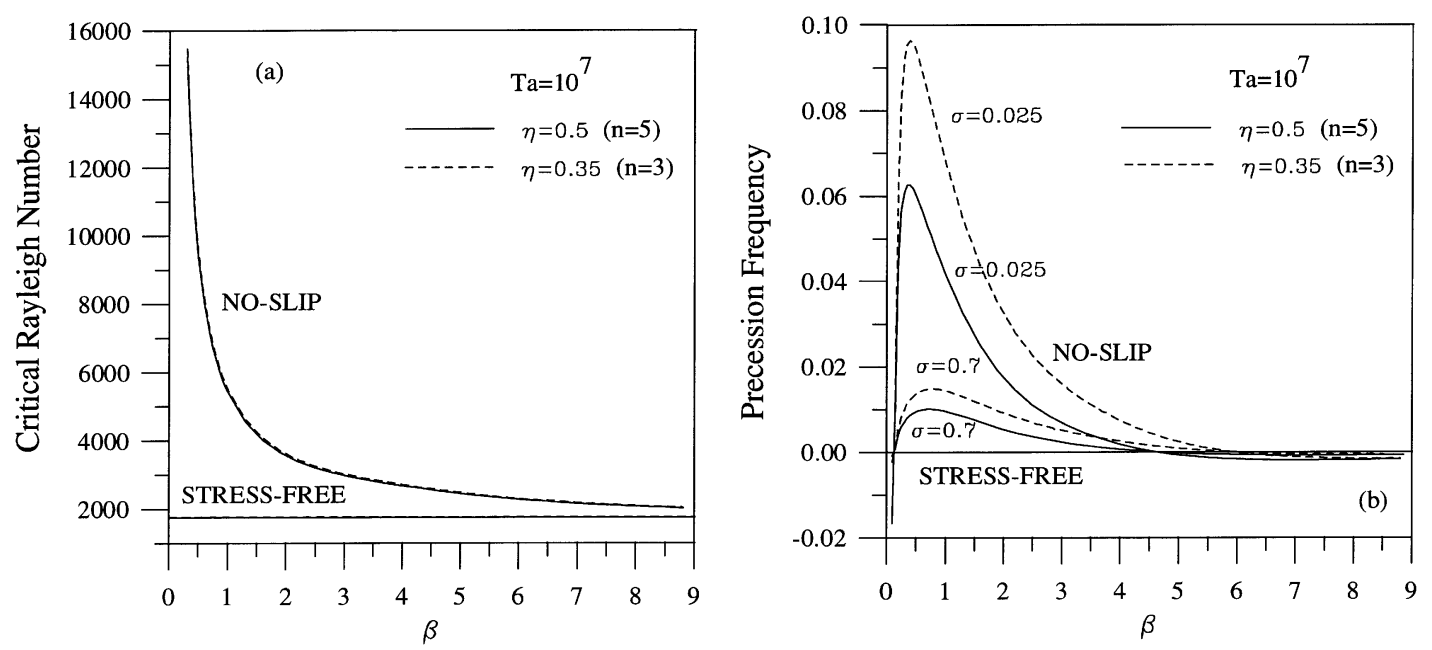

Fig. 5. (a) The critical Rayleigh number and (b) the corresponding precession frequency in viscous units as functions of the (inverse) aspect ratio, $\beta$, for stress-free and no-slip boundary conditions on the lids. The parameters are $\mathrm{Ta}=10^{7}$, $\eta=0.35,0.5$ and $\sigma=0.025,0.7$. The value of $n$ indicates the dominant azimuthal wave number for each $\eta$.
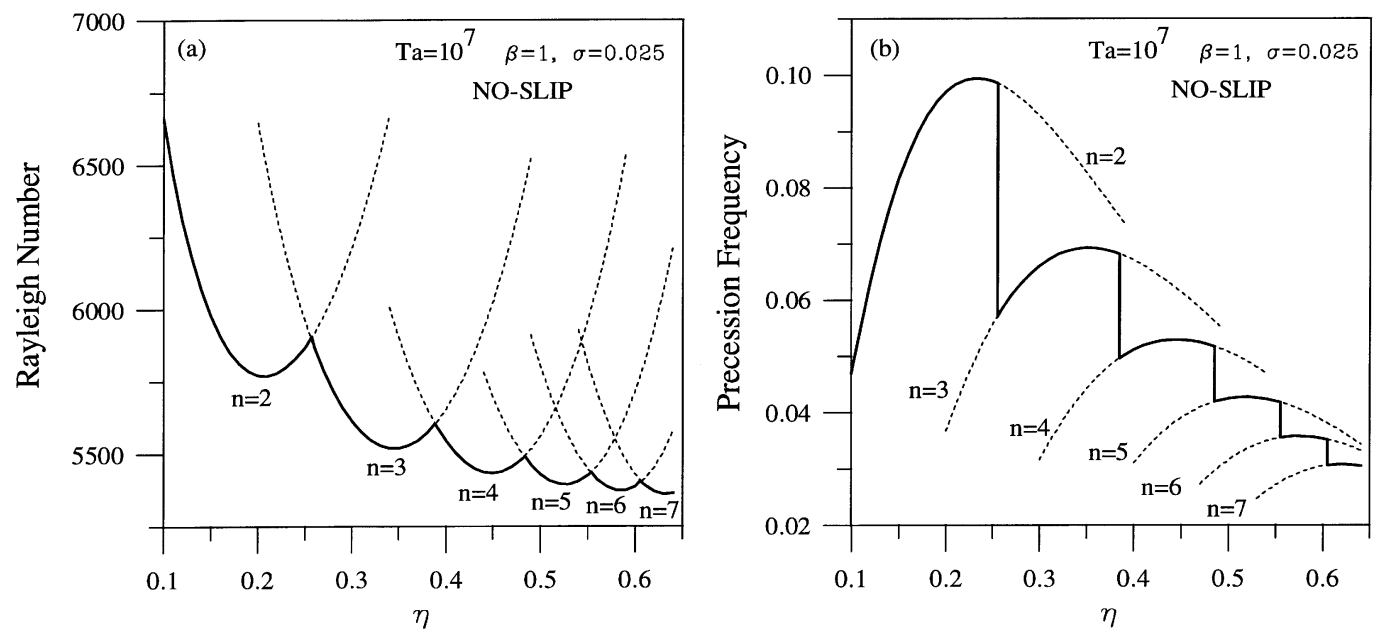

Fig. 6. (a) The critical Rayleigh number and (b) the corresponding precession frequency in viscous units as functions of the radius ratio, $\eta$, for no-slip boundary conditions on the lids and $\mathrm{Ta}=10^{7}$.

in Fig. 6a the selected wave numbers are likely to be constant in larger ranges of Rayleigh numbers with no-slip boundary conditions than with stress-free ones. Note that in the limit $\eta \rightarrow 1 \mathrm{Ra}_{\mathrm{c}}$ and $\omega_{\mathrm{c}}$ approach, as expected, the results for thermal convection in a rectangular rotating channel and that here the azimuthal waves travel in a retrograde sense $\left(\omega_{\mathrm{c}}>0\right)$; this is a consequence of the moderate aspect ratio $(\beta=1)$. As predicted by Busse (1970) and confirmed in Fig. 7 the dominant modes of the no-slip case at high rotation rates are practically independent of the axial position except near the top and bottom lids, where very thin Ekman boundary layers are present. These small 


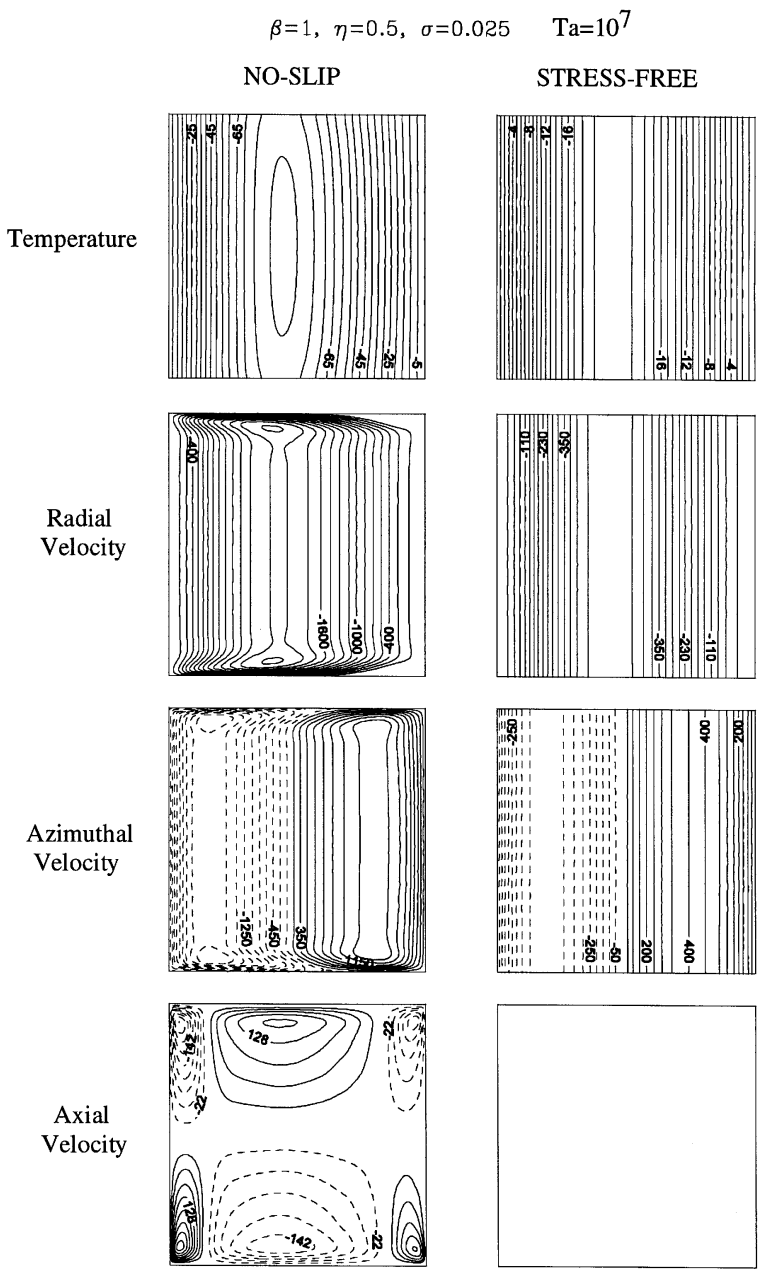

Fig. 7. Contour plots of the velocity components and temperature perturbation showing the axial structure of the dominant convection modes in an $(r, z)$ plane for stress-free and no-slip boundary conditions on the lids. The parameters are $\mathrm{Ta}=10^{7}$, $\beta=1, \eta=0.5$ and $\sigma=0.025$.

regions, where the Taylor-Proudman constraint breaks down, control the dynamics of the system (Greenspan, 1968) and are responsible for the large critical Rayleigh number needed for the onset of the convection when $\beta$ is small (see Figs. 5 and 6). To compensate for the viscous action in these layers a small vertical velocity close to the lids must be present, as shown in the figure. The development of these Ekman layers and the transition from fully three-dimensional convection cells to the almost two-dimensional columns can be seen in Fig. $8 \mathrm{a}$ and $8 \mathrm{~b}$, where curves of $\mathrm{Ra}_{\mathrm{c}}$ and $\omega_{\mathrm{c}}$ as a function of the Taylor number are presented. Fig. 8a reveals a gradual change in the slope of the Rayleigh number curve in the range of Taylor numbers where the Ekman layers begin to develop. Furthermore, as shown in Fig. 8b, this change coincides with the change from prograde precession $\left(\omega_{\mathrm{c}}<0\right)$ to retrograde $\left(\omega_{\mathrm{c}}>0\right)$ precession. This transition depends on $\eta$, but typically takes place 

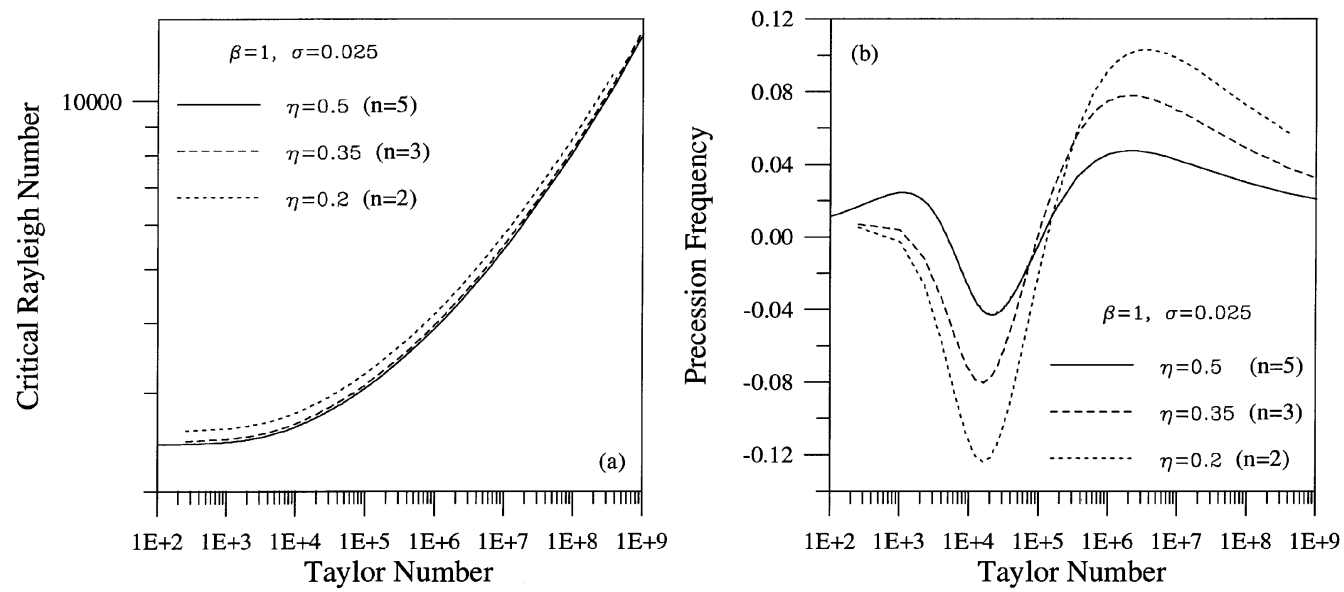

Fig. 8. (a) The critical Rayleigh number and (b) the corresponding precession frequency in viscous units as functions of the Taylor number, Ta, for no-slip boundary conditions on the lids and several radius ratios. The parameters are $\beta=1$, $\sigma=0.025$. The value of $n$ indicates the dominant azimuthal wave number for each $\eta$.

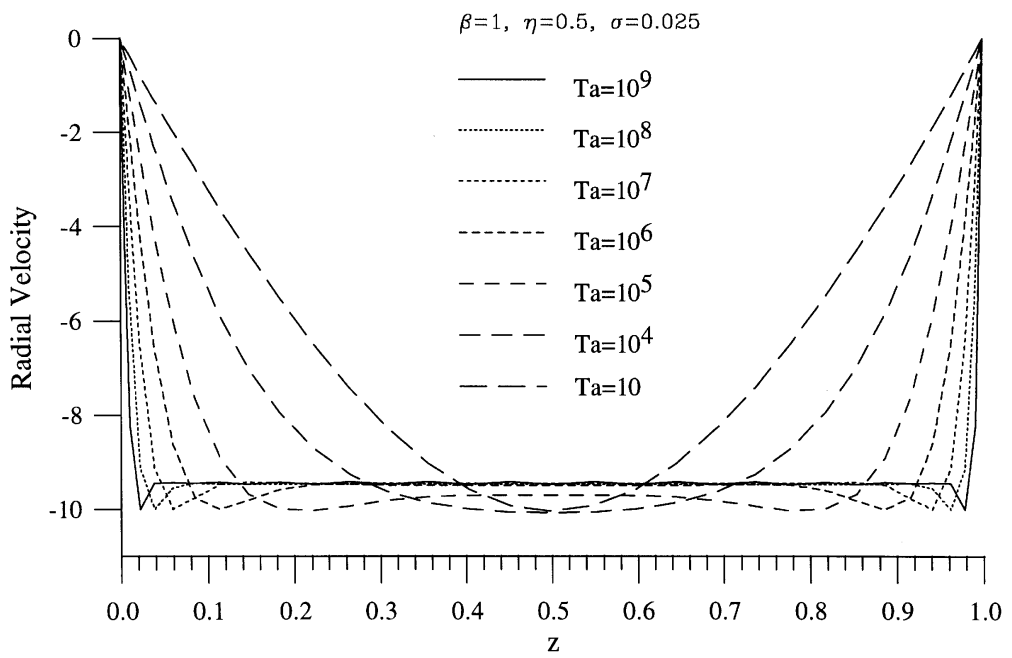

Fig. 9. The evolution of the Ekman boundary layers with increasing Taylor number Ta for $\beta=1, \eta=0.5$ and $\sigma=0.025$.

between $\mathrm{Ta}=10^{3}$ and $10^{5}$. For constant $\beta \lesssim 4.6$, the range of prograde frequencies increases with decreasing $\eta$, but $\omega_{\mathrm{c}}$ always becomes positive (retrograde precession) near $\mathrm{Ta}=10^{5}$ indicating that an almost two-dimensional state is achieved. For larger $\beta$ the asymptotic precession frequency remains prograde. Support for this argument is also provided in Fig. 9, plotted for $\eta=0.5$. At $\mathrm{Ta} \approx 10^{4}$ the radial velocity component starts to flatten out at mid-height and by $\mathrm{Ta}=10^{5}$ the Ekman structure is already well developed. From this figure we can estimate the thickness $\delta$ of the Ekman layer, and find that $\delta \approx 3 \times \mathrm{Ta}^{-0.24}$, thereby confirming the expected relation $\delta \propto \Omega^{-1 / 2}$. This agreement also confirms the accuracy of the calculations at high rotation rates. In order to show the evolution with increasing 


$$
\beta=1, \eta=0.5, \sigma=0.025
$$

(a)

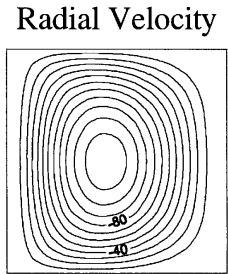

(b)

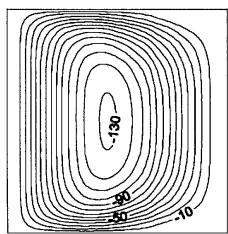

(c)

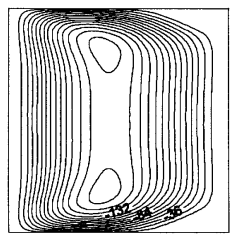

(d)

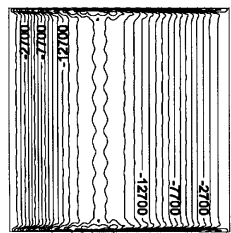

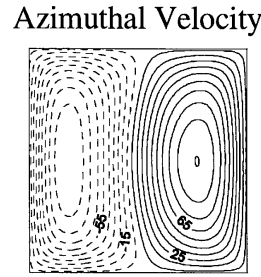
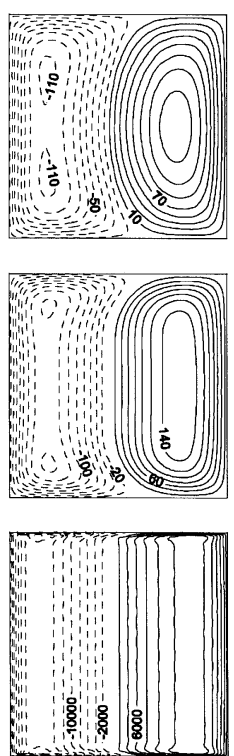
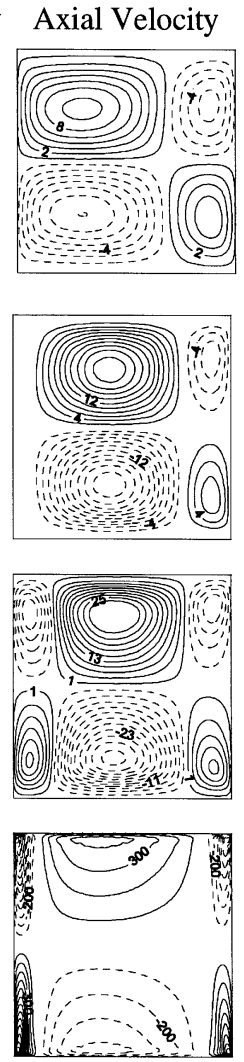

Temperature
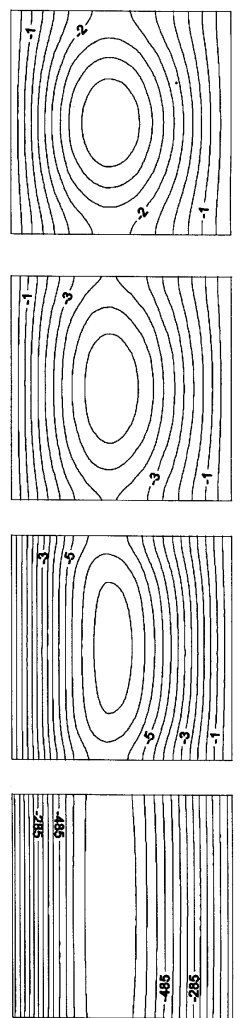

Fig. 10. Contour plots of the velocity components and temperature perturbation in an $(r, z)$ plane showing the formation of the Ekman boundary layers by increasing the Taylor number for $\beta=1, \eta=0.5$ and $\sigma=0.025$. (a) $\mathrm{Ta}=10^{3},(\mathrm{~b}) \mathrm{Ta}=10^{4}$, (c) $\mathrm{Ta}=10^{5}$, (d) $\mathrm{Ta}=10^{9}$.

Taylor number even more graphically, we show in Fig. 10 contour plots of the velocity components and the temperature perturbation in an $(r, z)$ plane corresponding to a section of a Taylor-Proudman column for several different Taylor numbers, all drawn on the same scale to indicate the relative order of magnitude of the variables. For example, at $\mathrm{Ta}=10^{9}$, the vertical velocity is more than 30 times smaller than the other velocity components or the dimensionless temperature perturbation. It is this change in the mode structure that is responsible for the change in the direction of precession with increasing Taylor number (see Fig. 8b). Interestingly, a similar oscillation in the precession frequency is also found with stress-free boundary conditions at top and bottom (Greed and Zhang, 1996), provided one restricts attention to three-dimensional modes, indicating that a similar transition of the vertical mode structure takes place with increasing rotation rate in this case as well.

Finally, in Fig. 11 we have analyzed the degree of influence of the type of boundary conditions on the ends of the annulus by imposing the Robin boundary conditions

$$
(1-\gamma) \frac{\partial^{2} w}{\partial z^{2}}+\gamma \frac{\partial w}{\partial z}=0 \quad \text { on } z=0, \beta
$$



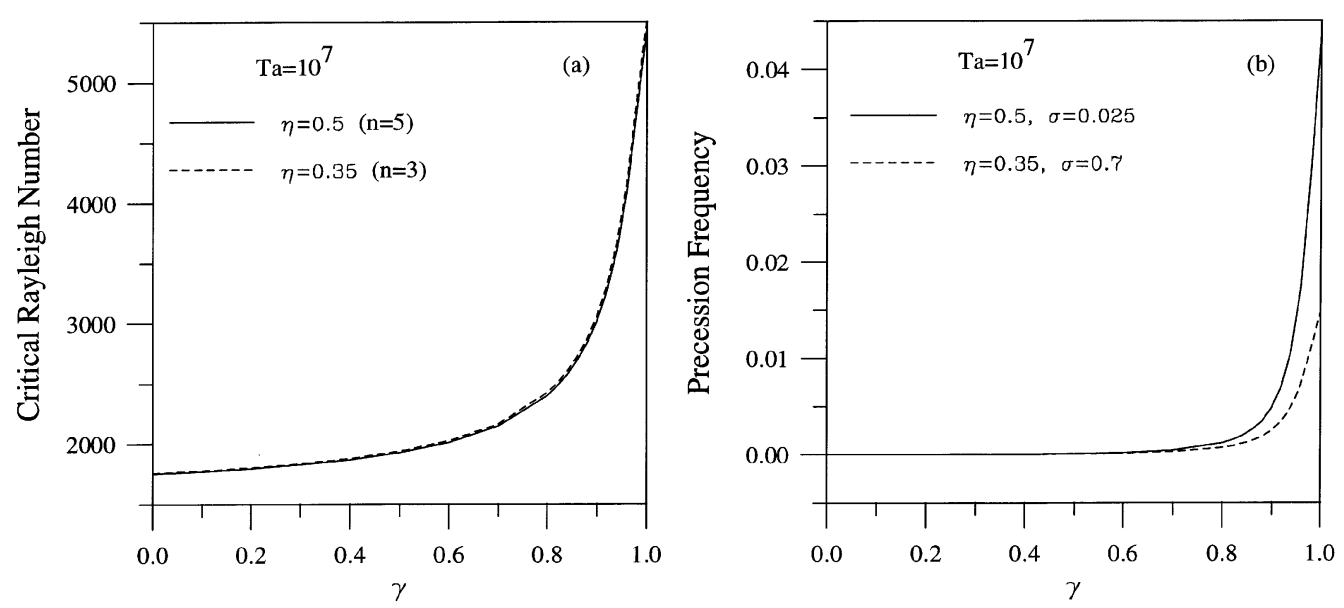

Fig. 11. (a) The critical Rayleigh number and (b) the corresponding precession frequency in viscous units as functions of the homotopy parameter $\gamma$ for two values of $\eta$. The parameters are Ta $=10^{7}, \beta=1$. The value of $n$ indicates the dominant azimuthal wave number for each $\eta$.

Here $\gamma$ is a homotopy parameter that provides a continuous connection between stress-free conditions $(\gamma=0)$ and no-slip boundary conditions $(\gamma=1)$. Note that the critical Rayleigh number and precession frequency approach those for the no-slip conditions $(\gamma=1)$ only for $\gamma>0.8$. For smaller values of $\gamma$ the requisite Ekman boundary layers do not develop, at least for the parameter values considered. However, the precession frequency is nonzero for all $\gamma>0$, i.e., for all non-Neumann boundary conditions at the lids.

\section{Conclusions}

In this paper we have shown that for sufficiently high Taylor numbers convection in a rotating, radially heated annulus with radial gravity takes the form of nearly two-dimensional Taylor-Proudman columns. These columns form with increasing Taylor number via a continuous lengthening of the three-dimensional states that are present in moderate aspect ratio annuli at small rotation rates. The columns are stationary in the rotating frame if stress-free boundary conditions are employed at the top and bottom of the annulus, but precess if the more realistic no-slip boundary conditions are used instead. The direction of this precession depends on the aspect ratio of the annulus. If the annulus is sufficiently long the precession is prograde, while in a shorter annulus the precession is typically prograde at intermediate Taylor numbers only but becomes retrograde at high Taylor numbers. The precession originates in the Ekman boundary layers at the top and bottom which form in the presence of no-slip boundaries. In these boundary layers the Taylor-Proudman theorem breaks down and the Coriolis force cannot be balanced by the pressure gradient. We have focused on low Prandtl number fluids for which the precession is so slow that the first bifurcation is nearly steady and therefore almost independent of the Prandtl number. Such solutions are characteristic of low viscosity fluids rather than those with high thermal diffusivity. However, for fixed Taylor number there is always a transition to the latter case once the Prandtl number becomes sufficiently small. Conversely, for a 
fixed but low Prandtl number the system behaves like a high thermal diffusivity one at small Taylor numbers but a low viscosity one at high Taylor numbers. Associated with the transition between these two regimes is the topology change revealed graphically in Fig. 10.

Our high Taylor number results are in qualitative agreement with the large Taylor number asymptotics carried out by Zhang and Greed (1997) for a fluid with uniform internal heating who showed that

$$
\mathrm{Ra}_{\mathrm{c}}=\mathrm{Ra}_{0}+C \frac{\mathrm{Ta}^{1 / 4}}{\beta},
$$

where $\mathrm{Ra}_{0}$ is the critical Rayleigh number for the stress-free problem and $C$ is a Prandtl-number independent constant. Zhang and Greed also noted that the precession frequency vanished in the high Taylor number limit but did not determine its parameter dependence at finite Ta. It is important to realize that for such high Taylor number columns the flow outside the boundary layers is potential and hence that nonzero helicity is confined to the boundary layers. Such flows would therefore be very inefficient at generating magnetic fields by the dynamo mechanism.

\section{Acknowledgements}

This work was supported by DGICYT under grant PB94-1216 and a CEPBA computer time grant.

\section{References}

Alonso, A., Net, M., Knobloch, E., 1995. On the transition to columnar convection. Phys. Fluids 7, 935-940.

Azouni, M.A., Bolton, E.W., Busse, F.H., 1986. Convection driven by centrifugal buoyancy in a rotating annulus. Geophys. Astrophys. Fluid Dyn. 34, 301-317.

Busse, F.H., 1970. Thermal instabilities in rapidly rotating systems. J. Fluid Mech. 44, 441-460.

Busse, F.H., Carrigan, C.R., 1974. Convection induced by centrifugal buoyancy. J. Fluid. Mech. 62, 579-592.

Fein, J.S., Pfeffer, R.L., 1976. An experimental study of the effects of Prandtl number on thermal convection in a rotating, differentially heated cylindrical annulus of fluid. J. Fluid Mech. 75, 81-112.

Greed, G.T., Zhang, K., 1996. Two and three dimensional convection in a rotating annulus. Geophys. Astrophys. Fluid Dyn. 82, 23-34.

Greenspan, H.P., 1968. The Theory of Rotating Fluids. Cambridge University Press, Cambridge.

Hide, R., Mason, P.J., 1975. Sloping convection in a rotating fluid. Adv. Phys. 24, 47-100.

Knobloch, E., 1994. Bifurcations in rotating systems. in: Proctor, M.R.E., Gilbert, A.D. (Eds.), Lectures on Solar and Planetary Dynamos. Cambridge University Press, Cambridge, pp. 331-370.

Marqués, F., Net, M., Massaguer, J.M., Mercader, I., 1993. Thermal convection in vertical cylinders: a method based on potentials of velocity. Comput. Meth. Appl. Mech. Eng. 110, 157-169.

Read, P.L., Bell, M.J., Johnson, D.W., Small, R.M., 1992. Quasi-periodic and chaotic flow regimes in a thermally driven, rotating fluid annulus. J. Fluid Mech. 238, 599-632.

Zhang, K., Greed, G.T., 1997. Convection rolls controlled by Ekman boundary layers: an asymptotic theory and numerical solutions. Preprint. 